Portland State University

PDXScholar

\title{
Sound Propagation, Reflection, and Its Relevance to Ultrasound Imaging
}

Thomas Allen

Portland State University

Alex Chally

Portland State University

Bradley Moser

University of New England

Ralf Widenhorn

Portland State University, ralfwidenhorn@pdx.edu

Follow this and additional works at: https://pdxscholar.library.pdx.edu/phy_fac

Part of the Physics Commons

Let us know how access to this document benefits you.

\section{Citation Details}

Allen, T., Chally, A., Moser, B., \& Widenhorn, R. (2019). Sound Propagation, Reflection, and Its Relevance to Ultrasound Imaging. The Physics Teacher, 57(3), 134-137.

This Article is brought to you for free and open access. It has been accepted for inclusion in Physics Faculty Publications and Presentations by an authorized administrator of PDXScholar. Please contact us if we can make this document more accessible: pdxscholar@pdx.edu. 


\section{Sound Propagation, Reflection, and Its Relevance to Ultrasound Imaging}

Thomas Allen, Alex Chally, Bradley Moser, and Ralf Widenhorn

Citation: The Physics Teacher 57, 134 (2019); doi: 10.1119/1.5092466

View online: https://doi.org/10.1119/1.5092466

View Table of Contents: https://aapt.scitation.org/toc/pte/57/3

Published by the American Association of Physics Teachers

\section{ARTICLES YOU MAY BE INTERESTED IN}

WATER ROLL

The Physics Teacher 57, 131 (2019); https://doi.org/10.1119/1.5092463

Kinetic Friction: Time Upward and Downward

The Physics Teacher 57, 142 (2019); https://doi.org/10.1119/1.5092469

Two Beakers, Five E's, Twenty Pennies, and Archimedes' Principle

The Physics Teacher 57, 138 (2019); https://doi.org/10.1119/1.5092468

A Variable-Mass Snowball Rolling Down a Snowy Slope

The Physics Teacher 57, 150 (2019); https://doi.org/10.1119/1.5092471

Playground Physics: What Happens When Trying a $360^{\circ}$ Swing?

The Physics Teacher 57, 146 (2019); https://doi.org/10.1119/1.5092470

Geometrical, Fresnel, and Fraunhofer Regimes of Single-Slit Diffraction

The Physics Teacher 57, 176 (2019); https://doi.org/10.1119/1.5092479

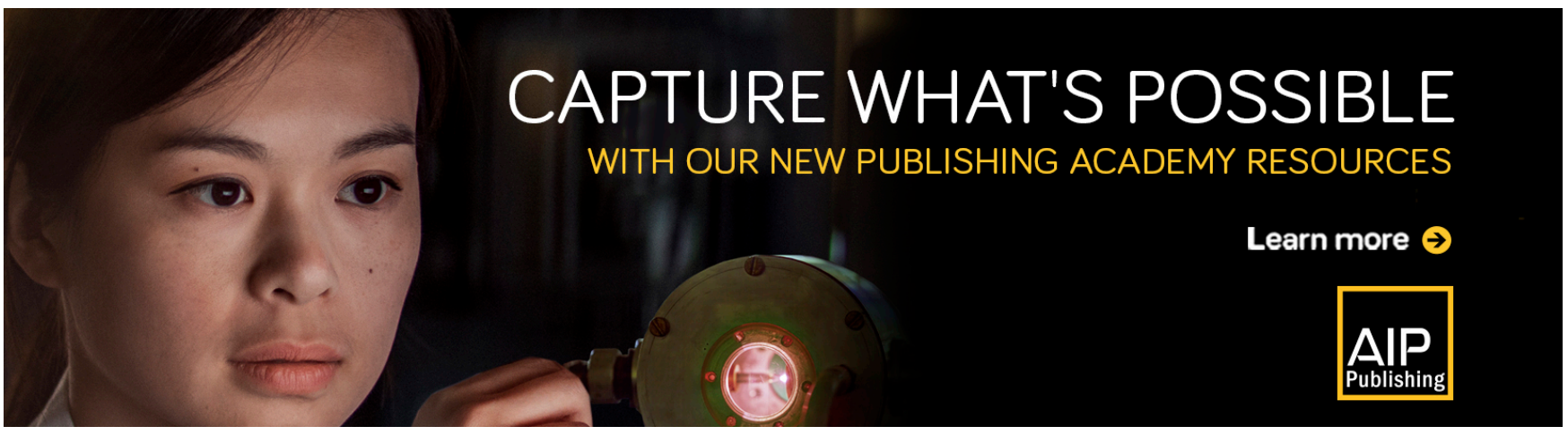




\section{Sound Propagation, Reflection, and Its Relevance to Ultrasound Imaging}

Thomas Allen and Alex Chally, Portland State University, Portland, OR

Bradley Moser, University of New England, Biddeford, ME

Ralf Widenhorn, Portland State University, Portland, OR

$\mathrm{T}$ The labs presented here build on a simple speed of sound activity and models medical ultrasound imaging by demonstrating how multiple reflections propagate in a closed system. A short sound pulse is emitted into a pipe that is closed at one end and contains one or more partially reflecting surfaces within the pipe. The variety of reflections and transmissions that occur can be measured with a microphone at the pipe entrance.

We used white PVC pipes ( $4 \mathrm{~m}$, schedule 40,2 -in diameter) and cut them into five pieces with lengths $0.4 \mathrm{~m}, 0.6 \mathrm{~m}$, $0.8 \mathrm{~m}, 1 \mathrm{~m}$, and $1.2 \mathrm{~m}$ (Fig. 1), cut to a precision of $\pm 1.6 \mathrm{~mm}$. We connected the pipes with couplings and modified some couplings to cause partial reflections, by covering $1 / 4,1 / 2$, or $3 / 4$ of the coupling opening. For easiest adoption, use repair coupling rather than a standard coupling. If standard couplings are used we recommend boring them out so that the pipes can easily be connected and disconnected.

This activity requires a source to create a sound pulse as well as a receiver and logging/visualization software. A finger snap is the traditional source of sound, but some students struggle to produce consistently strong, short pulses. Sound

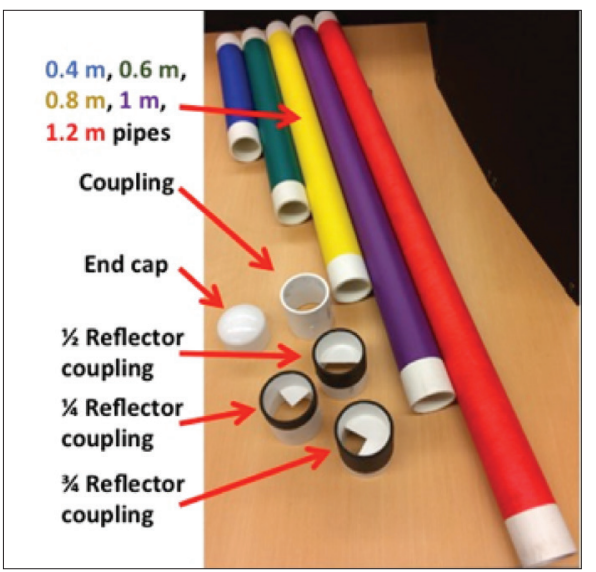

Fig. 1. Pipes, couplings, and end cap used in the experiment. One can assemble various length combinations with partial reflectors. Students can analyze the timing and magnitude of the reflected signals. editors with speakers, or even headphones, can provide this consistency. For instance, we implemented this activity in a teaching lab with multiple lab groups and had good results using Audacity to generate a tone in the $1000-$ to $4000-\mathrm{Hz}$ range, a compact digital stereo amplifier (Lvpin 2020A+), and custom-made small speakers. For the results in this paper we used Audacity to generate a 1-ms tone at $2000 \mathrm{~Hz}$, and headphones as a speaker. This pulse is short enough in duration to minimize the overlap of echoes in short pipes or pipes with multiple reflectors. Students can investigate how changing the pulse duration impacts the reflection data. Additionally, a sound editor that can produce a series of pulses will allow students to investigate the importance of selecting an appropriate pulse repetition rate. We used the commercially available Vernier microphone and Logger Pro data logging/visualization software to analyze the data obtained in the following experiments. The acquisition software was triggered to start recording when the sound pressure of the initial pulse exceeded a preset pressure level, which may vary depending on the sound source. We also set the sampling rate at 10,000 samples per second and the duration of recording at $0.1 \mathrm{~s}$.

The pipes and couplings can be assembled a number of ways (Fig. 2). Each pipe and coupling setup increases the complexity of the travel paths of the sound waves to mimic the reflection of ultrasound from internal body structures by allowing for reflections from multiple interfaces at different distances.

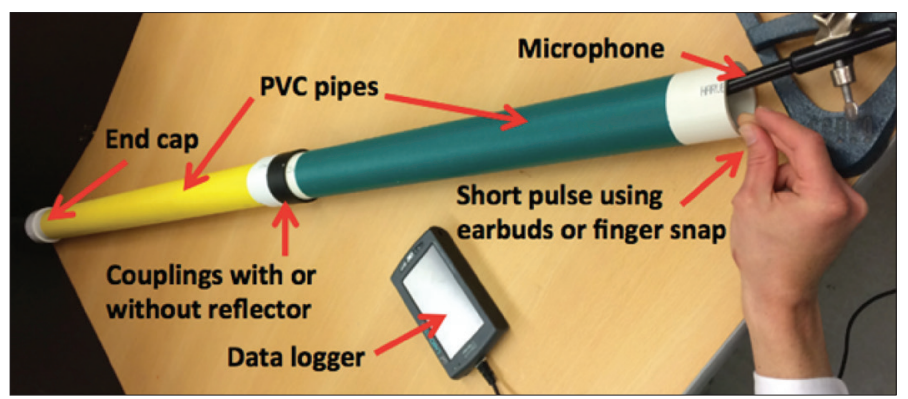

Fig. 2. Experimental setup: The sound is emitted from a finger snap (or earbuds) and travels along the pipe. The sound reflects off the end cap and off the coupling if it contains a reflector.

\section{Use in the classroom}

After completing the lab exercises, students will be able to:

- Measure the speed of sound in air.

- Identify the paths that sound waves travel based on multiple reflections.

- Establish a connection between the time-of-flight signal received from multiple interfaces in the apparatus and the reflections from multiple body surfaces measured in ultrasound imaging.

This lab activity can be divided into a series of four experiments. The lab can appear early in the curriculum on sound waves in undergraduate introductory physics or a more specialized physics in medicine course. ${ }^{2}$ We use the lab in conjunction with curriculum that is relevant for pre-health students and videos by biomedical experts. ${ }^{3-5}$

The lab requires no advanced terminology or concepts of the physics of sound. Mathematically, it only uses the constant velocity model, $x=v^{\star} t$. Conceptually, the activity can be used to introduce the ideas of reflection and transmission off of multiple surfaces. Experimentally, the activity can be used to 


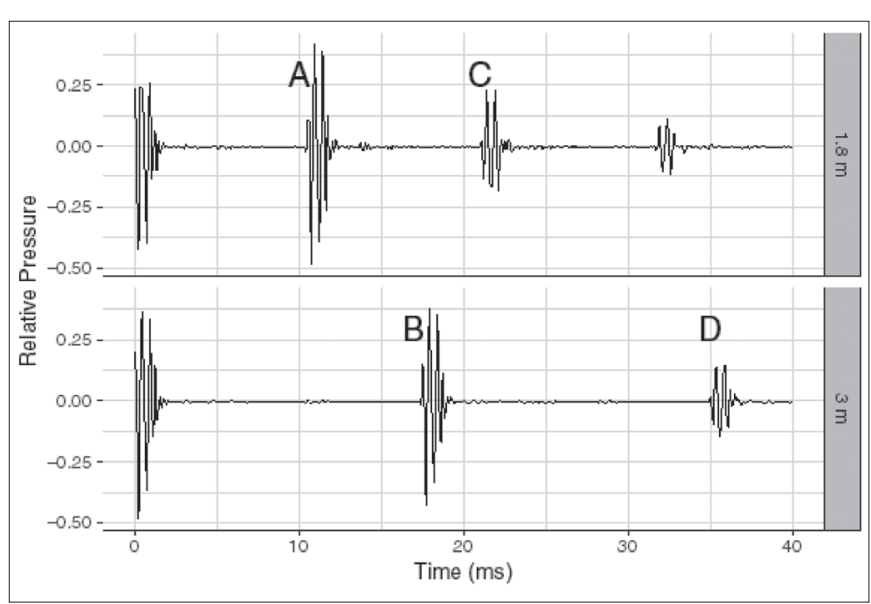

Fig. 3. Sound pulse reflections using earbuds as sound source in the 3-m and the 1.8-m pipes. The first peak at $t=0$ in each plot is the initial sound pulse. Each successive pulse corresponds to a reflected pulse. The labeled times correspond to $(A)$ the first reflection off the end of the 1.8-m pipe, $(B)$ the first reflection off the 3-m pipe, (C) the second reflection off the 1.8-m pipe, and (D) the second reflection off the 3-m pipe.

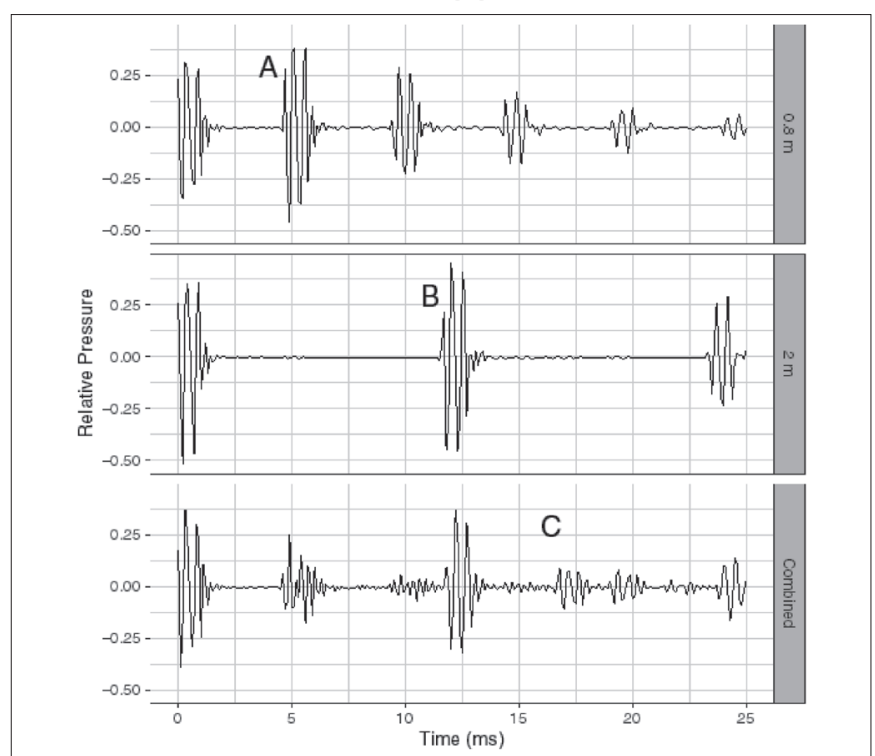

Fig. 4. Sound pulse reflections from three different pipe arrangements using earbuds as sound source: a pipe $0.8 \mathrm{~m}$ in length, a pipe $2 \mathrm{~m}$ in length, and a pipe $2 \mathrm{~m}$ in length with a partial reflector at the junction between the $0.8-\mathrm{m}$ and $1.2-\mathrm{m}$ pipes. The labeled times correspond to (A) the first reflection off the $0.8-\mathrm{m}$ pipe and the first reflection off the partial reflector located at $0.8 \mathrm{~m}$ in the 2-m pipe, (B) the first reflection off the end of the 2-m pipe, and (C) where there is superposition due to waves traveling different path lengths.

establish the speed of sound and determine the location of the partial reflections.

\section{Experiment 1}

The first experiment is a basic measurement of a sound wave's echo, which appears readily in the literature. ${ }^{6-9}$ The sound wave propagates down a pipe, reflects from either a closed or open end, and is measured with a microphone at the pipe entrance. This experiment demonstrates reflection of sound and establishes the speed of sound in air. The various pipe lengths and couplings allow for easily changing the travel distance.

First, students explore the reflections of sound waves for different length pipes. Figure 3 shows these reflections. These data will be used as a reference for when reflective couplings are included later. However, the initial sound pulse and its first reflection are not the only signal measured. Sound amplitude peaks return at equal intervals of time. These represent subsequent reflections; the first echo reflects from the open end of the pipe, travels to the closed end, and reflects a second time. This repeats with decreasing amplitude for subsequent reflections. Students can calculate the speed of sound or use the theoretical speed of sound to calculate the distance of the reflection to the sound source. The distance measurement would be the information used in ultrasound imaging. The calculated distances can be compared to the pipe length. From the data in Fig. 3, for example:

First reflection 3-m pipe:

Reflection distance $=\left(340 \mathrm{~m} / \mathrm{s}{ }^{\star} 17.5 \mathrm{~ms}\right) / 2=2.98 \mathrm{~m}$

Third reflection 1.8-m pipe:

Reflection distance $=(340 \mathrm{~m} / \mathrm{s} * 31.9 \mathrm{~ms}) /(2 * 3)=1.81 \mathrm{~m}$

\section{Experiment 2}

The second experiment builds on the first one by introducing a partially reflecting surface within the pipe. These reflectors partially reflect and partially transmit the initial sound wave. We have tried multiple ways of producing partial reflections. The best results are from plastic semi-circles glued to the couplings (Fig. 1). However, this limits reflectors to being placed at the junction of two pipes. A surprising richness emerges in the time-of-flight data as the sound waves can take many different round-trip paths (Fig. 4). Each path can be identified with a little sleuthing.

Figure 4 shows three data sets from this experiment. The first data plotted are reflections from a 0.8 - $\mathrm{m}$ long pipe with an end cap. Four reflections are distinctly visible. The next data plotted are reflections from a $2-\mathrm{m}$ long pipe with an end cap, assembled by connecting a $0.8-\mathrm{m}$ and $1.2-\mathrm{m}$ long pipe. Again, four reflections, spaced further apart, are visible. These two data sets serve as reference for the final data, which show the reflections that occur when a $1 / 2$ partial reflector is placed at the junction of the pipes. This allows partial transmission into the second pipe (replicating the 2-m pipe) and partial reflection (replicating the $0.8-\mathrm{m}$ pipe). Therefore, the amplitude peaks should match both of the previous data sets, as is seen. The first and second reflections from the 0.8 - $\mathrm{m}$ length pipe (5.0 $\mathrm{ms}$ and $10.0 \mathrm{~ms}$ ) and 2-m length pipe ( $12 \mathrm{~ms}$ and $24 \mathrm{~ms}$ ) are visible, although the amplitude is noticeably reduced for the first reflection and nearly extinguished after the second. This is because the amplitude is split by reflection/transmission each time the partial reflector is encountered.

The first significant feature is that the two reflecting surfaces can be distinguished-a reflection from the partial 


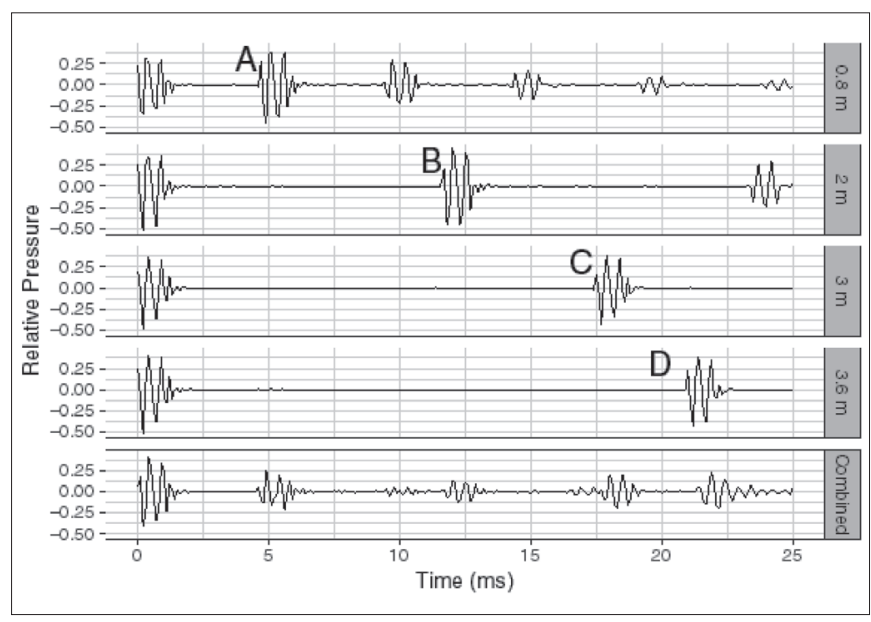

Fig. 5. Reflections from three different pipe arrangements using earbuds as sound source: a pipe $0.8 \mathrm{~m}$ in length, a 2-m pipe, a 3-m pipe, and a 3.6-m pipe. Also plotted is the 3.6- $\mathrm{m}$ pipe with $1 / 2$ and $3 / 4$ reflectors (combination of $0.8 \mathrm{~m}$ and $1 / 2$ reflector and $1.2 \mathrm{~m}$ and $1 / 2$ reflector and $1 \mathrm{~m}$ and $3 / 4$ reflector and $0.6 \mathrm{~m}$ ). Note that the combined data show reflections from all three reflectors with the same timing but different amplitudes as the previous combination. The labeled times correspond to $(A)$ the first reflections off an $0.8-\mathrm{m}$ pipe, (B) the first reflection off a 2-m pipe, (C) the first reflection off a 3-m pipe, and (D) the first reflection off of a 3.6-m pipe.

reflector and a reflection from the back reflector. This is crucial in ultrasound imaging technology; for instance, some percentage of an ultrasound beam reflects from the front surface of an organ within the body while some is transmitted through the organ and reflects from the back surface.

The second feature is the new peaks and enhanced peaks that appear in the partial reflector data set. The peak at $17.5 \mathrm{~ms}$, labeled as $\mathrm{C}$ in the bottom panel of Fig. 4, is a new peak and cannot be explained by multiple reflections from a $0.8-\mathrm{m}$ or $2-\mathrm{m}$ length pipe. Instead it is a result of paths that include the partial reflector. There are two different possible paths that the sound wave can travel to contribute to this peak. In one path, the original pulse reflects from the partial reflector, returns to the entrance of the pipe, reflects from the open end, travels the entire length of the pipe after transmitting through the partial reflector, reflects off the end, and finally arrives at the open end again (after a second transmission through the partial reflector). In another path, the original pulse is transmitted through the partial reflector, travels the entire length of the pipe, reflects off the end, is transmitted through the entire pipe again, reflects off the open end, and finally reflects off the partial reflector. Therefore, the total amplitude of the sound peak at $17.5 \mathrm{~ms}$ is a superposition of two paths. It may be instructive to ask students to identify some peaks that could be due to the superposition in their experimental setup and then consider what possible paths could contribute to those peaks.

\section{Experiment 3}

The third experiment extends the second one by adding additional reflectors in the pipe (Fig. 5). This experiment draws the analogy to ultrasound medical imaging where

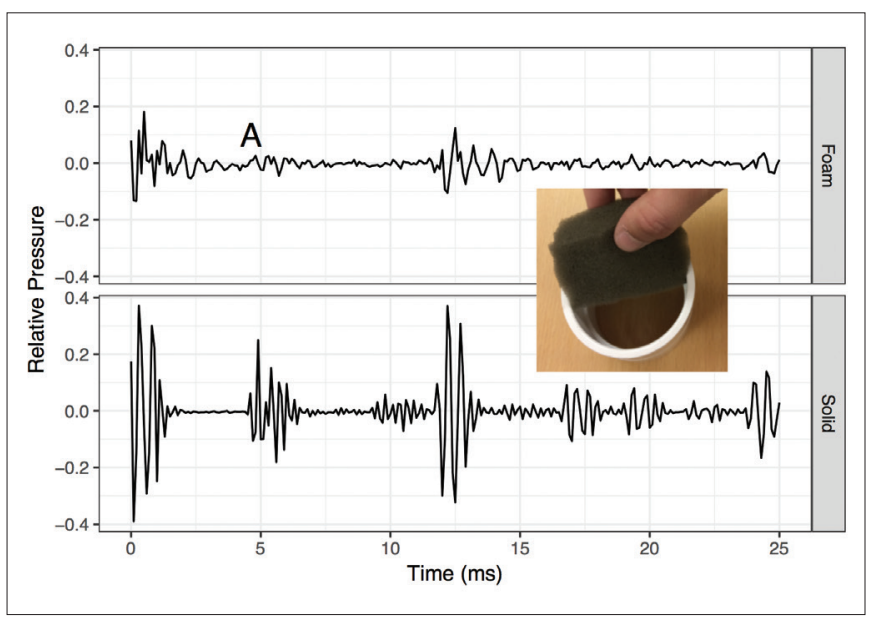

Fig. 6. Reflections in 2-m pipes, composed out of 0.8-m and 1.2-m pipes, with two different $1 / 2$ reflectors, using a finger snap as the sound source. The top panel shows the resulting reflections when a foam reflector is used and the bottom panel shows the resulting reflections when a solid reflector is used. The time labeled (A) corresponds to the first reflections off of both reflectors. Note the reduced amplitude of the reflection from the foam reflector. The inset shows the foam partial reflector used in experiment 4 . The foam reflector can be placed in an empty coupling.

piezoelectric transmitters and receivers register multiple reflected signals to reconstruct a grayscale image.

For this experiment we use four different pipes and three partial reflectors (two $1 / 2$ reflectors and one $3 / 4$ reflector).

Figure 5 shows four sets of data using four different lengths of pipe. The $0.8-\mathrm{m}$ pipe with an end cap, the $2-\mathrm{m}$ pipe $(0.8$ $\mathrm{m}$ and $1.2 \mathrm{~m})$ with an end cap, and the $3-\mathrm{m}$ pipe $(0.8 \mathrm{~m}$ and $1.2 \mathrm{~m}$ and $1 \mathrm{~m}$ ) with an end cap, respectively. The fifth data set is the combined data showing the $3.6 \mathrm{~m}(0.8 \mathrm{~m}$ and $1.2 \mathrm{~m}$ and $1 \mathrm{~m}$ and $0.6 \mathrm{~m}$ ) pipe with partial reflectors included. It is evident from Fig. 5 that a signal from each reflector can be recovered. Similar to experiment 2 , the secondary reflections from the 0.8- $\mathrm{m}$ and 2- $\mathrm{m}$ tube can be observed; however, higher order reflections are too attenuated to be observed.

One artifact that reduces the image quality in ultrasound is reverberation. This artifact appears when the ultrasound beam encounters two closely spaced strong reflecting surfaces. Reverberation can be experimentally explored by placing two reflectors close together within the pipe.

\section{Experiment 4}

For the fourth experiment, the plastic reflectors are replaced with foam reflectors (inset of Fig. 6). The foam piece is inserted into a coupling. This experiment simulates reflection off of materials with absorbing properties, such as human tissue or air in medical ultrasound imaging. We use the same pipes as experiment 2 with the foam reflector placed at their connection. Although the peak amplitudes of the reflections occur at the same time, the magnitude of the amplitude of the reflection from the foam reflector is reduced compared with the plastic reflector (Fig. 6). 


\section{Conclusion}

We present experiments expanding upon a typical speed of sound laboratory to model medical ultrasound imaging by including reflectors at different locations along a closed pipe and by allowing the material properties of these reflectors to be changed. Each experiment in this series builds upon the previous experiments to allow students to explore a simple model of ultrasound imaging. The length of the pipes, the location, and the number of reflectors can all be changed to add variety to the measurements made in these experiments.

\section{References}

1. Audacity is free open-source cross-platform audio software for multi-track recording and editing, http://www.audacityteam. org/.

2. G. R. Van Ness and Ralf Widenhorn, "Engaging the community through an undergraduate biomedical physics course," Am. J. Phys. 80, 1094-1098 (Dec. 2012).

3. Elliot Mylott, Ellynne Kutschera, Justin C. Dunlap, Warren Christensen, and Ralf Widenhorn, "Using biomedically relevant multimedia content in an introductory physics course for life science and pre-health students," J. Sci. Educ. Technol. 25 (2), 222-231 (April 2016).
4. Warren Christensen, James K. Johnson, Grace R. Van Ness, Elliot Mylott, Justin C. Dunlap, Elizabeth A. Anderson, and Ralf Widenhorn, "Developing and assessing curriculum on the physics of medical instruments," CBE Life Sci. Educ. 12 (2), 250-61 (June 2013).

5. Physics in Biomedicine, YouTube, https://www.youtube.com/ user/PhysicsinBiomedicine/.

6. M. G. Raymer and S. Micklavzina, "Demonstrating sound impulses in pipes," Phys. Teach. 33, 183-185 (1995).

7. L. Kasper, P. Vogt, and C. Strohmeyer, "Stationary waves in tubes and the speed of sound," Phys. Teach. 53, 52-53 (Jan. 2015).

8. R. D. Knight, B. Jones, and S. Field, College Physics: A Strategic Approach, 3rd ed. (Pearson, Boston, 2015), Chap. 15.

9. "Speed of Sound in a Snap," Vernier, https://www.vernier.com/ innovate/speed-of-sound-in-a-snap/, or "Measuring the Speed of Sound with an Xplorer GLX," PASCO scientific, https:// www.pasco.com/support/technical-support/technote/techIDlookup.cfm?TechNoteID=564. A web search will provide further possible lab experiment write-ups from faculty at various universities.

Portland State University, Portland, OR 97207-0751; thallen@pdx.edu

\title{
And the Survey Says...
}

\author{
Susan C. White, Column Editor \\ American Institute of Physics \\ Statistical Research Center \\ College Park, MD 20740; swhite@aip.org
}

\section{How much do physics faculty members earn?}

We surveyed faculty members in two- and four-year colleges and universities in the United States to find out. We contacted faculty members teaching physics in two-year colleges and in departments that grant degrees in physics. We heard back from about 1600 faculty members. Among the data we gathered were salaries. We now have an interactive online tool that provides faculty salaries based on a number of factors including:

- Type of institution

- Highest physics degree offered in the department

- $9 / 10$ months, $11 / 12$ months, or by course

- Degree earned

- Gender
- Full- or part-time

- Location of institution (state or national average)

- Academic rank (including adjunct)

- Postdoc completion

- Includes HBCUs

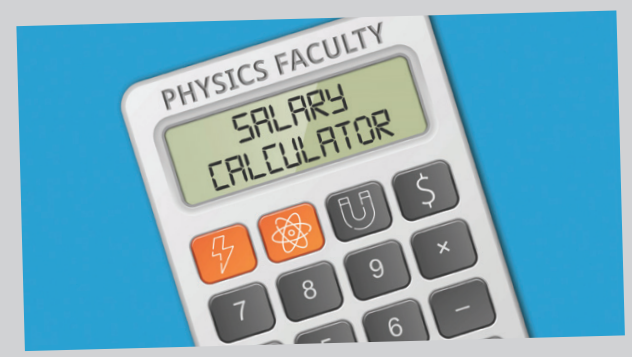

Please go to www.aip.org/statistics/salary-calculator to use the tool to find out what physics faculty members earn.

If you have any questions, please contact us. Susan White works in the Statistical Research Center at the American Institute of Physics. She can be reached at swhite@aip.org. DOI: $10.1119 / 1.5092467$ 